\title{
Modeling of dendrite growth from undercooled nickel melt: sharp interface model versus enthalpy method
}

\author{
A Kao ${ }^{1}, \mathrm{~L}$ V Toropova $^{2}, \mathrm{D}$ V Alexandrov ${ }^{2}, \mathrm{G}$ Demange $^{3}$ and P \\ K Galenko ${ }^{2,4}$ \\ ${ }^{1}$ Centre for Numerical Modelling and Process Analysis, University of Greenwich, Old \\ Royal Naval College, Park Row, London SE10 9LS, UK \\ 2 Department of Theoretical and Mathematical Physics, Laboratory of Multi-Scale \\ Mathematical Modeling, Ural Federal University, Ekaterinburg, 620000, Russian \\ Federation \\ ${ }^{3}$ GPM, CNRS-UMR 6634, University of Rouen Normandy, 76801, Saint Ètienne Du \\ Rouvray, France \\ ${ }^{4}$ Friedrich-Schiller-Universität-Jena, Physikalisch-Astronomische Fakultät, 07743 \\ Jena, Germany \\ E-mail: A.Kao@greenwich.ac.uk \\ E-mail: Dmitri.Alexandrov@urfu.ru
}

October 2019

\begin{abstract}
The dendritic growth of pure materials in undercooled melts is critical to understanding the fundamentals of solidification. This work investigates two new insights, the first is an advanced definition for the two-dimensional stability criterion of dendritic growth and the second is the viability of the enthalpy method as a numerical model. In both cases, the aim is to accurately predict dendritic growth behavior over a wide range of undercooling. An adaptive cell size method is introduced into the enthalpy method to mitigate against 'narrow-band features' that can introduce significant error. By using this technique an excellent agreement is found between the enthalpy method and the analytic theory for solidification of pure nickel.
\end{abstract}

\section{Introduction}

Experimentation and theoretical modeling with the solidification of nickel and nickelbased alloys revealed a lot of information about the structure of melts and crystals forming under different driving forces and influences of various fields [1-3]. One of the first experimental estimations of solidification kinetics was conducted using melt flux and levitation techniques [4-8] on the basis of which some estimations of speed limited growth of crystals were made [9]. The roles of solute trapping and solute drag in rapidly solidifying diluted nickel-based alloys were investigated by Eckler et 
al. $[10,11]$. The effect of convective flow on growth kinetics was also quantitative for Nidendrites [12]. Using an original technique for experimental measurements, Schwarz et al. formulated the mechanism of grain refinement [13] as well as presented measurements of diffusive speed and diffusion coefficients $[14,15]$. The effect of microgravity on crystal growth [16] and microstructure of undercooled melts [17,18] was measured in an electro-magnetic facility. These experiments used the containerless methods for sample processing [1,2] and provide a deep undercooling in melts, in which forced convection may drastically influence the growth kinetics when the growth velocity is comparable to the flow speed $[12,19,20]$. A comparative analysis of crystallization kinetics and crystal microstructure was given to clarify the role of external fields, such as gravitational [16], alternating electromagnetic [19] and static magnetic fields [21,22].

Theoretical modeling and predictions were made for crystals of nickel and nickelbased alloys using the atomistic and mesoscopic models. In molecular dynamics (MD) simulations, one of the central tasks was to obtain the thermodynamic and kinetic properties of solid-liquid interfaces (see Ref. [23] and references therein). In some cases, the interface energy (tension) can be well found from the laboratory experiments using, for instance, the method of nucleation statistics [24]. However, for nickel, a kinetic phenomenon at the interface such as the "attachment-detachment of particles" cannot be reproduced due to its opaqueness. Therefore, MD simulations are an important source of kinetic data, in particular, for the growth coefficients of different crystallographic faces of nickel $[25,26]$. Such data provides the phase-field models with the necessary material properties to allow the growth and analysis of the structure of nickel dendrites [27,28]. The linear and non-linear behaviors of the interface velocity for a different interface undercooling in MD simulations [25,26] were also obtained as a benchmark for the kinetic equations of growth that follow, in particular, from the phase-field models [29].

In addition to this, transformations in multi-component melts based on nickel under the influence of buoyancy-driven melt convection were modeled using the phase-field method by Apel and Steinbach [30]. Special attention has been paid to the comparison of the sharp interface model and the phase-field methods [31,32]. The sharp interface model possesses the zero thickness of the solid-liquid interface and it is based on the Stefan-type theoretical approach. This model determines the first condition connecting the dendrite tip velocity, the dendrite tip diameter, and the melt undercooling. The second condition is defined by the selection theory, which enables to find a stable solution of heat and mass transfer equations in the vicinity of dendritic tip region (see, for details, [33-37]). A less commonly known enthalpy method has been successfully used to model the dendritic growth in undercooled melts [38]. The method has been used to allow not only the prediction of kinetics but also some microstructural details of crystalline (in particular, dendritic) patterns [22,39].

In all of these studies with the enthalpy method only specific undercoolings have been investigated and the method has not been tested as an accurate predictor across a wide range of undercoolings. One advantage of the enthalpy method is that the interface thickness is not explicitly required as part of the formulation, however 
for implementation purposes an interface consisting of a single computational cell is required. This introduces the so called 'narrow-band feature' error in curvature calculation, which is related to the amount of local information about the morphology and cannot be mitigated through mesh refinement [38]. Using smoothed differentials can somewhat alleviate the problem, however additional techniques to minimise this error are required. This work presents a novel technique that exploits the self-symmetry of dendrite tips, where a dimensionless form of the curvature and an adaptive cell size method is used to show that the enthalpy method can be used across a wide range of undercoolings. Therefore, the main goal of the present article is to investigate consistency between the well established sharp interface model and the enthalpy method. A sharp interface model that includes the selection mode for arbitrary growth Péclet numbers [40] is compared with the numerical enthalpy method for the two-dimensional tip velocity and radius of dendrites.

\section{Sharp interface model}

\subsection{Undercooling balance}

The dendrite tip diameter $\rho$ and its growth velocity $V$ represent the main parameters of crystal growth. The total undercooling balance connects the melting temperature $T_{m}$ of a single-component liquid and the far-field temperature $T_{\infty}$ as $\Delta T=T_{m}-T_{\infty}$ and introduces the first model equation, which consists of several contributions:

$\Delta T=\Delta T_{T}+\Delta T_{R}+\Delta T_{K}$

Here $\Delta T_{T}$ is the thermal contribution, $\Delta T_{R}=2 d_{0} T_{Q} / R$ is the two-dimensional undercooling due to the Gibbs-Thomson effect, $T_{Q}$ is the adiabatic temperature, $d_{0}$ is the capillary constant, and $\Delta T_{K}=V / \mu_{k}$ is the kinetic undercooling, where $\mu_{k}$ stands for the kinetic coefficient.

The thermal contribution $\Delta T_{T}$ can be written out using the Ivantsov function $\mathrm{Iv}_{T}$, which describes the temperature field around the the growing steady-state dendrite of a parabolic form:

$\Delta T_{T}=T_{Q} \operatorname{Iv}_{T}\left(P_{g}\right)$,

where the Ivantsov function $\mathrm{Iv}_{T}$

$\operatorname{Iv}_{T}\left(P_{g}\right)=P_{g} \exp \left(P_{g}\right) \int_{1}^{\infty} \frac{\exp \left(-P_{g} \eta^{\prime}\right)}{\sqrt{\eta^{\prime}}} d \eta^{\prime}$

depends on the growth Péclet number $P_{g}=\rho V /\left(2 D_{T}\right)\left(D_{T}\right.$ stands for the thermal diffusivity).

Finally, the total undercooling balance (1) can be reformulated in a parametric form of $P_{g}$ as [41]:

$\Delta T=\Delta T_{T}\left(P_{g}\right)+\frac{4 d_{0} T_{Q}}{\rho\left(P_{g}\right)}+\frac{2 D_{T} P_{g}}{\mu_{k} \rho\left(P_{g}\right)}$. 
Equation (4) represents the undercooling balance, the solution of which determines only the product $\rho V$ as a function of the undercooling $\Delta T$, and does not give the information about the dependencies $\rho(\Delta T)$ and $V(\Delta T)$ in a separate form. For this reason, a second equation providing a selection criterion is needed. This criterion is found from the solvability theory [42-45].

\subsection{Solvability criterion}

Consider a two-dimensional parabolic dendrite growing in a single-component undercooled liquid. In the case of low anisotropy of kinetics and surface energy, the temperature distribution is in close proximity to the parabolic Ivantsov solution, which describes a steady-state dendritic growth. Pelcé and Bensimon [42] (see also [43, 44]) showed that this statement leads to the microscopic solvability condition representing an approximate analytical solution of the linearized heat transfer equation at the parabolic surface of an Ivantsov dendrite. This leads to the solvability condition:

$\int_{-\infty}^{\infty} G\left[X_{0}(l)\right] Y_{m}(l) d l=0, \quad Y_{m}(l)=\exp \left[i \int_{0}^{l} k_{m}\left(l_{1}\right) d l_{1}\right]$,

where $G$ designates the curvature operator, $X_{0}(l)$ is a continuum of solutions from which the dependence of the marginal wavenumber mode $k_{m}(l)$ can be derived, and $i$ is the imaginary unit. Note that the solution $G\left[X_{0}(l)\right]$ is orthogonal to the imposed perturbation $Y_{m}(l)$ that provides a stable mode.

To obtain the marginal wavenumber $k_{m}$ entering in the solvability integral (5), a linear stability analysis should be carried out [41]. In this case, the marginal mode of the wavenumber $k_{m}$ (see [44] for details), is determined by the cubic equation:

$$
k_{m}^{3}=\frac{V \exp (i \theta)}{2 d(\theta) D_{T}} k_{m}-\frac{i V \sin \theta}{2 D_{T}} k_{m}^{2}+\frac{V^{2} \cos \theta \exp (i \theta)}{4 d(\theta) D_{T}^{2}}+\frac{i V \tilde{\beta}(\theta) \sin \theta}{d(\theta) T_{Q}} k_{m}^{2},
$$

where $\theta$ is the angle between the normal to the dendrite interface and its growth direction. The capillary length $d$ in the case of $n$-fold symmetry of the crystal is expressed as $d(\theta)=d_{0}[1-\beta \cos (n \theta)]$.

Substituting the analytical solution of the cubic equation (6) into the solvability integral (5), yields the stability criterion [40] in the form:

$\sigma^{*}=\frac{2 d_{0} D_{T}}{\rho^{2} V}=\frac{d_{0}}{\rho P_{g}}=\frac{\sigma_{0} \beta^{7 / 4}}{\left(1+a_{1} \sqrt{\beta} P_{g}\right)^{2}}$,

where $\sigma_{0}$ and $a_{1}$ are the constants. This criterion describes the broad range of possible Péclet numbers. Note that expression (7) transforms to the low Péclet number stability criterion previously discussed in many studies (see, among others, [42, 43, 46-48]).

Equation (7) represents the unified selection criterion which gives a combination of $\rho$ and $V$ for the thermal dendritic growth in single-component systems without 
convection $\ddagger$. Considering the undercooling balance (4) and the selection criterion (7), a pair of the most important parameters of primary solidification, $\rho$ and $V$, at a given undercooling $\Delta T$ can be obtained.

\section{Numerical modelling using an enthalpy based method}

The transient numerical model uses an enthalpy method for dendritic growth, based on the work of Voller [38]. Building from the initial work of Tacke and co-workers [49,50], Voller took the classical sharp interface model and through the introduction of the order parameter, $f$, formulated a diffuse interface method based on enthalpy. $f$ represents the liquid fraction, where $f=1$ is fully liquid and $f=0$ is fully solid. Intermediate values of $f$ represent the interface where computational cells are solidifying. One of the key differences between this formulation and more traditional phase-field methods is that the interface thickness is not explicitly defined nor is a key parameter in the governing equations $[51,52]$. To relate $f$ to enthalpy, the volumetric enthalpy $H$ is defined as the sum of latent heats:

$H=c_{p} T+f L$.

The conservation of enthalpy is given by

$\frac{\partial H}{\partial t}=\nabla \cdot(K \nabla T)$.

The thermal conductivity $K$ is assumed to be constant. The interface is undercooled to the temperature $T^{i}$ :

$T^{i}=T_{m}-\frac{\Gamma(\theta)}{L} T_{m} \kappa$,

where the surface energy anisotropy takes the form $\gamma=d_{0}\left(1+\epsilon_{4} \cos 4 \theta\right)$ and therefore the surface stiffness is given by

$\Gamma(\theta)=\gamma+\frac{\partial^{2} \gamma}{\partial \theta^{2}}=d_{0}\left(1-15 \epsilon_{4} \cos 4 \theta\right)$.

Curvature is taken as the divergence of the normal, which in terms of liquid fraction is $\kappa=\nabla \cdot \frac{\nabla f}{|\nabla f|}$.

Voller converted this equation set into a dimensionless system and then discretized it onto a Cartesian grid. The same approach is used here and so for brevity, this approach is not repeated, however, there are two important aspects of the numerical approach that are necessary for the improvements in this presented work. The first is a calculation of curvature, where for a cubic mesh i.e. the cell lengths are equal, $\Delta x=\Delta y$, equation (12) can be written as

$\kappa=\frac{1}{\Delta x} \frac{f_{y y} f_{x}^{2}+f_{x x} f_{y}^{2}-2 f_{x} f_{y} f_{x y}}{\left(f_{x}^{2}+f_{y}^{2}\right)^{\frac{3}{2}}}$,

$\ddagger$ The criterion (7) was also derived for the dendrite growth with convection in the work [40]. The tests of the criterion with convection were made in the works [21,22], in which again dendritic crystallization was analyzed at arbitrary Péclet numbers. 
where

$f_{x}=\Delta x \frac{\partial f}{\partial x}=\left(f_{i+1, j}-f_{i-1, j}\right) / 2$,

$f_{x x}=\Delta x^{2} \frac{\partial^{2} f}{\partial x^{2}}=\left(f_{i+1, j}-2 f_{i, j}+f_{i-1, j}\right)$

and similarily $f_{y}$ and $f_{y y}$ are the first and second derivatives in the $y$ direction multiplied by $\Delta x$ and $\Delta x^{2}$ respectively. The second aspect of the computational method is that liquid cells only become 'seeded' when neighboring cells fully solidify. This has the effect of creating a region where $0>f<1$, that is on the order of one computational cell. Therefore, while no explicit thickness is required by the formulation, in practice this creates an interface thickness that is proportional to the grid size. Consequently, this leads to the so-called narrow-band feature, which causes problems in calculating interfacial properties, namely curvature.

Therefore mesh refinement can actually increase errors, for example, the curvature is calculated locally and so if a large number of cells represent the tip radius then locally there may not be enough information to distinguish the curvature accurately. The narrow-band error leads to two effects, the curvature drives to zero if the interface locally appears flat or to a very large value if the interface has a 'corner'. This can cause the dendrite morphology to take on an unrealistic faceted 'diamond' like structures.

On the other hand, if the cell size is too large, errors associated with a coarse mesh are introduced, and in general, leads to an under prediction of curvature. For example, in the extreme case where the tip radius is less than a single cell then due to lack of resolution, the radius would be calculated as a single cell with an error on the order of $\Delta x$. This under-prediction of curvature should in principle lead to an over prediction of tip radius, but in practice, as the error essentially manifests in the GibbsThompson condition, it has an analogous effect of artificially lowering the anisotropy. This reduces the bias toward preferential growth orientations and allows the dendrite to grow secondary arms or for the tip to split, ultimately leading to competition between these new 'primaries' and therefore not an accurate representation of the system.

The coarse mesh and the narrow-band errors essentially introduce an upper and lower limit on the cell size for a given undercooling. However, due to the large disparity in characteristic length scales between low and high undercooled solidification, a criterion is required for selecting an appropriate value of $\Delta x$. This could be based on analytic theory, however, in the context of this investigation where testing and comparison to the analytic theory is a key aim, then the numerical solutions should not be dependent on it. Furthermore, the error in curvature will have a dependency on undercooling and subsequently, there will be undercooling dependent errors in both tip radius and velocity that are not straightforward to quantify. Instead, an approach has been developed that provides a similar relative error for all cases across the entire undercooling range.

The process begins by simply multiplying 13 through by $\Delta x$, such that the righthand side is no longer dependent on $\Delta x$ and the left-hand side becomes $\kappa \Delta x$. The morphological feature of interest is the tip radius, therefore by letting $\Delta x$ be chosen 
such that $\Delta x=(N+\epsilon) r_{0}$, where $N$ is the number of cells representing the tip radius and $\epsilon$ is the error from both the narrow-band and coarse mesh described previously, $\Delta x$ no longer appears. Given that $r_{0}=1 / \kappa$ equation (13) reduces to

$$
N+\epsilon=\frac{f_{y y} f_{x}^{2}+f_{x x} f_{y}^{2}-2 f_{x} f_{y} f_{x y}}{\left(f_{x}^{2}+f_{y}^{2}\right)^{\frac{3}{2}}} .
$$

This scaled curvature approach essentially removes physical length scales from the curvature calculation and for a given choice of $N$ only the local distribution of $f$ determines the curvature with a relative error. By assuming the dendrite tip morphology exhibits self-similarity across the entire undercooling range, the local distribution of $f$ should be somewhat similar irrespective of undercooling. However, as $r_{0}$ is unknown, $\Delta x$ also becomes an unknown. Therefore, an iterative approach is taken where $\Delta x$ adaptively changes based on the curvature (hence tip radius) calculations. If $N \Delta x$ is smaller than $r_{0}$ then $\Delta x$ is increased and similarly if $N \Delta x$ is larger than $r_{0}$ then $\Delta x$ is decreased. While this represents the physical size of the domain and the dendrite changing with time, at steady-state conditions $r_{0}, V$ and $\Delta x$ become constant.

The selection of $N$ depends on the behaviour of the coarse grid and narrowband feature errors. If $N$ is too small instabilities from the coarse grid error lead to unsteady solutions from oscillations of tip splitting and secondary branching. The narrow-band feature error, increases with $N$, however, due to the faceting-like nature this error introduces into the morphology, this error always under-predicts the tip radius. Therefore in this work, the optimal value of $N$ is assumed to be the smallest value that yields stable steady state solutions. It is worthwhile to note that this optimal of value of $N$ is not necessarily problem specific, but implementation specific and would depend on the stencil size and any other approximations to the differentials in the curvature calculation.

\subsection{Problem Setup and Parametric Study}

The discretized enthalpy formulation is solved on a square computational domain comprising $1200 \times 1200$ cells. Solidification occurs in the southwest corner of the domain. The far-field boundaries at the north and east are fixed to the bulk undercooled temperature. The south boundary is a symmetry boundary and the west boundary is of a Neumann type for both $T$ and $f$ with $\partial T / \partial x=\partial f / \partial x=0$.

To mitigate against influence from the boundary conditions, a moving mesh technique is used where the dendrite tip stays in the same relative position in the domain. In this work, this position is 100 cells in the $x$ direction. When the solidification front reaches this position all field variables are moved by one cell, for example $f_{i, j}=f_{i+1, j}$. On the west boundary information is lost, while on the east boundary far-field conditions are introduced into the last layer of cells.

A single simulation is used to conduct a parametric study across a wide range of undercooling starting at the highest undercooling. Using the previous steady-state 
values for $f$ and $T$ the next undercooling solution is preconditioned by modifying the temperature in all liquid cell values by the relative change in undercooling. This allows for the system to converge to a steady-state solution in a smaller number of time steps. Only the first undercooling solution is different, as initial conditions, including initial cell size, need to be specified. In this case, the entire domain is set to the bulk undercooled temperature and a small nucleus is placed in the southwest corner of the domain. As solidification advances, the tip reaches the $x$ position for the moving mesh. Then every time the mesh moves, the cell size and time interval are used to calculate the tip velocity, averaged curvature values over the solidification time of the cell are used to calculate tip radius. $\Delta x$ is then increased or decreased based on the tip radius, where the change is limited to $5 \%$ of its current value to ensure stability. If $\Delta x$ is within $1 \%$ of the current value of $r_{0} / N$ then $\Delta x$ is unchanged. $\Delta t=0.1 \Delta x^{2}$ is updated from $\Delta x$ and the process repeats until another cell solidifies and the mesh moves again. To further relax the convergence of the system the update of $\Delta x$ is carried out every time the mesh has moved twice. This also allows more time for the morphology of the tip to adapt to the new spacial scales as there is a temporal delay due to the release of latent heat and the subsequent thermal transport.

As the system approaches steady state, values for $\Delta x, \kappa$ and $V$ become constant, a solution is considered converged when the current values for $\Delta x, \kappa$ and $V$ vary by less than $0.1 \%$ to the previous 8 calculations of their respective values. The system is then preconditioned for the next undercooling and $\Delta x$ will increase to find a new equilibrium.

\section{Discussion}

A parametric study calculating steady-state tip velocity and radius for pure nickel was conducted over the range of $304 \mathrm{~K}$ down to $28 \mathrm{~K}$ undercooling. Figure 1 shows spot values normalized to the steady-state values, highlighting how the solution procedure progresses from one undercooling to the next, indicated by the discontinuities in the normalized variables.

Figure 2 shows the tip morphologies for 304, 189, 117, 73, 45 and $28 \mathrm{~K}$ undercooling. The circle in the figure represents the tip radius based on $N=8$. This result highlights how this adaptive cell size approach conforms to the self-similarity of the dendrite, where the real length scales between the highest and lowest undercooling results vary by almost two orders of magnitude.

Temperature contours are given in figures 3 and 4 for the highest and the lowest undercooling respectively with the insets focused on the tip undercooling. The bulk undercooling temperature has been blanked out in the figures, highlighting the furthest extent to which the thermal field expands into the domain. In the case of high undercooling, this is to around 150 cells of the dendrite, while in the low undercooled case the thermal field extends over 400 cells from the dendrite. With a domain of $1200 \times$ 1200 cells, the far-field boundaries do not influence the solution. The inset of the figures shows that this method can also handle large disparities in tip undercooling, shown here 


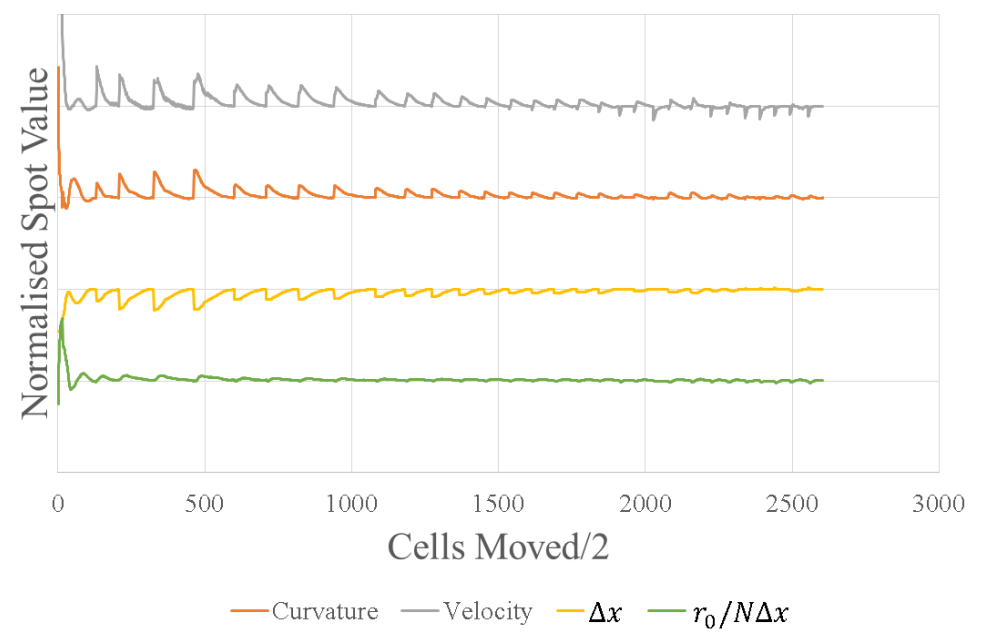

Figure 1. Typical normalised spot values for parametric study.

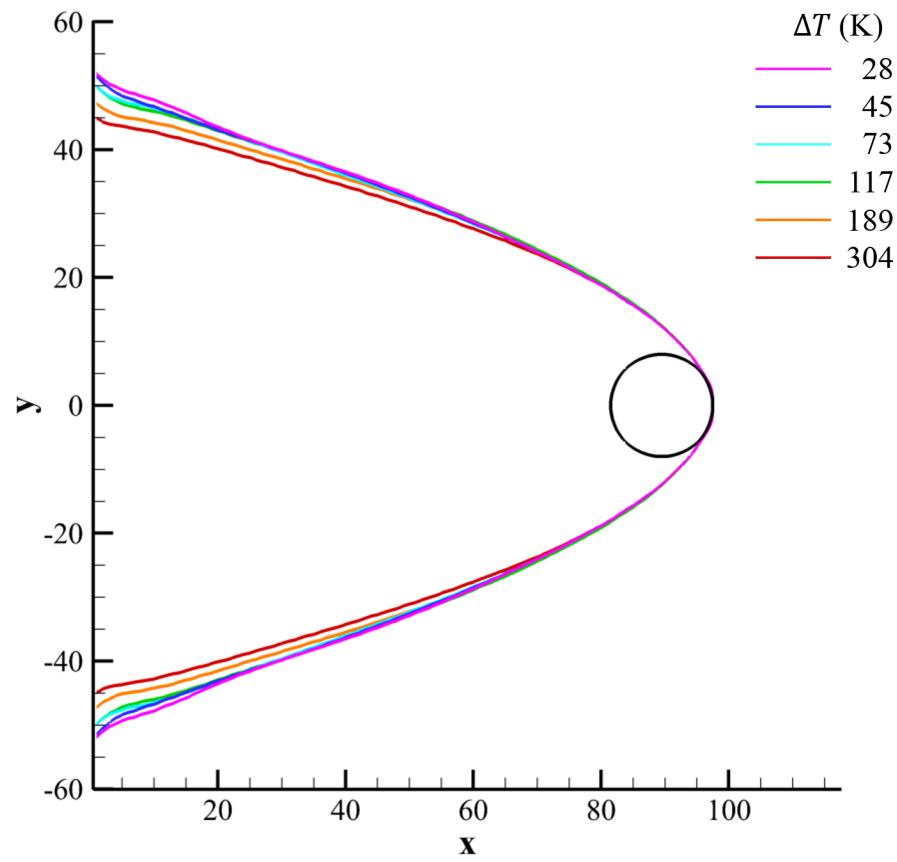

Figure 2. Dendritie morphology for various undercoolings. 


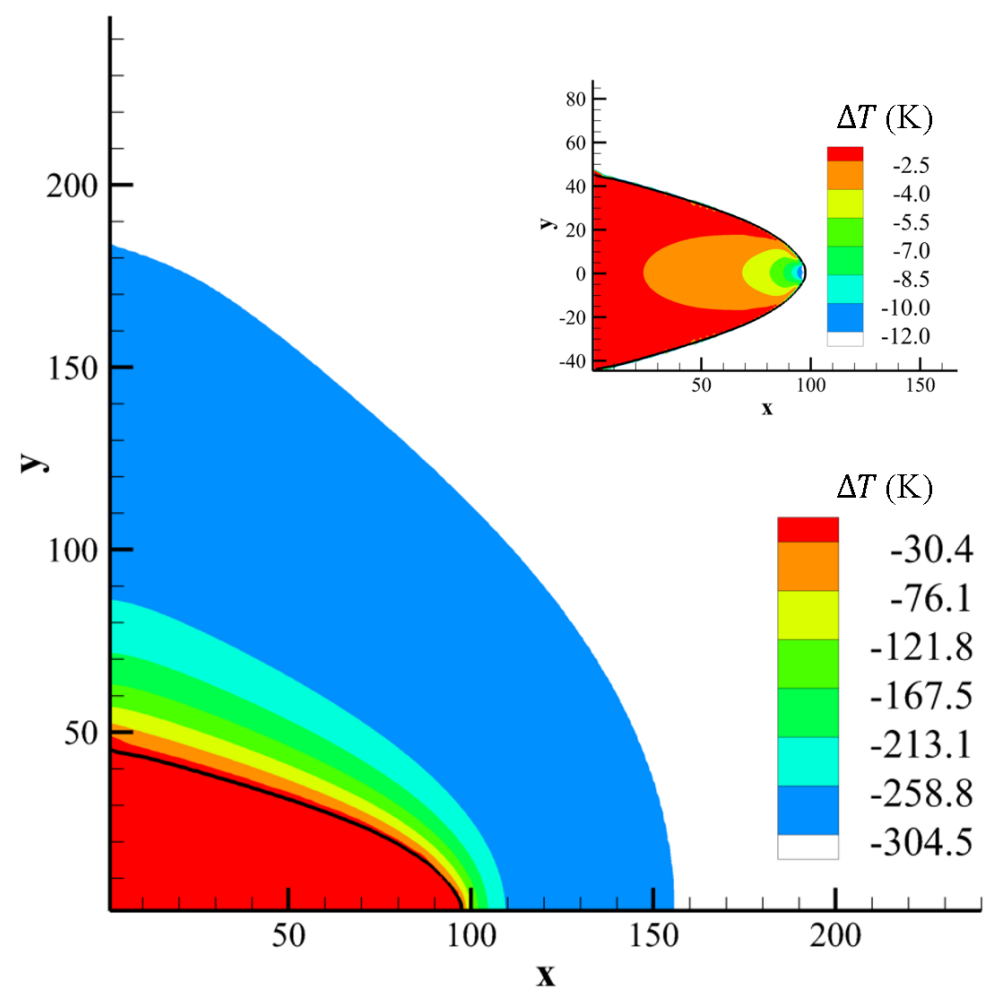

Figure 3. Thermal field for high, $304 \mathrm{~K}$, undercooling.

by two orders of magnitude difference ranging from $-10 \mathrm{~K}$ to $-0.1 \mathrm{~K}$. This is a direct consequence of the disparity in length scale, hence curvature which feeds into the GibbsThompson condition. The influence of the growth Péclet number can be clearly seen in both the main figure and the inset through the isotherms. In the high undercooled case, the isotherms take on an oblate shape, while in the low undercooled case they are more circular. Similarly in the insets, the isotherms in the high undercooled case are oblate and extended along the tip, while the low undercooled case they are again more circular.

These examples from the parametric study demonstrate two key points, the first is that as the enthalpy formulation does not explicitly require the interface thickness, a large disparity in cell size can be used, however, the trade-off is that coarse mesh and narrow-band errors can be introduced and are difficult to quantify. However, by exploiting the self-similarity of dendrite tip morphology and using an adaptive cell size that is proportional to a key morphological feature, in this case, tip radius, steadystate solutions for tip evolution can be obtained, where the relative error across a large undercooling range should be approximately the same.

A test of theoretical predictions obtained accordingly to the sharp interface model (Section 2) and numerical simulations carried out using the enthalpy method (Section $3)$ are illustrated in figures 5 and 6 . The theoretical curves for the dendrite tip velocity $V$ and its tip radius $\rho / 2$ as functions of the total undercooling $\Delta T$ are calculated from 


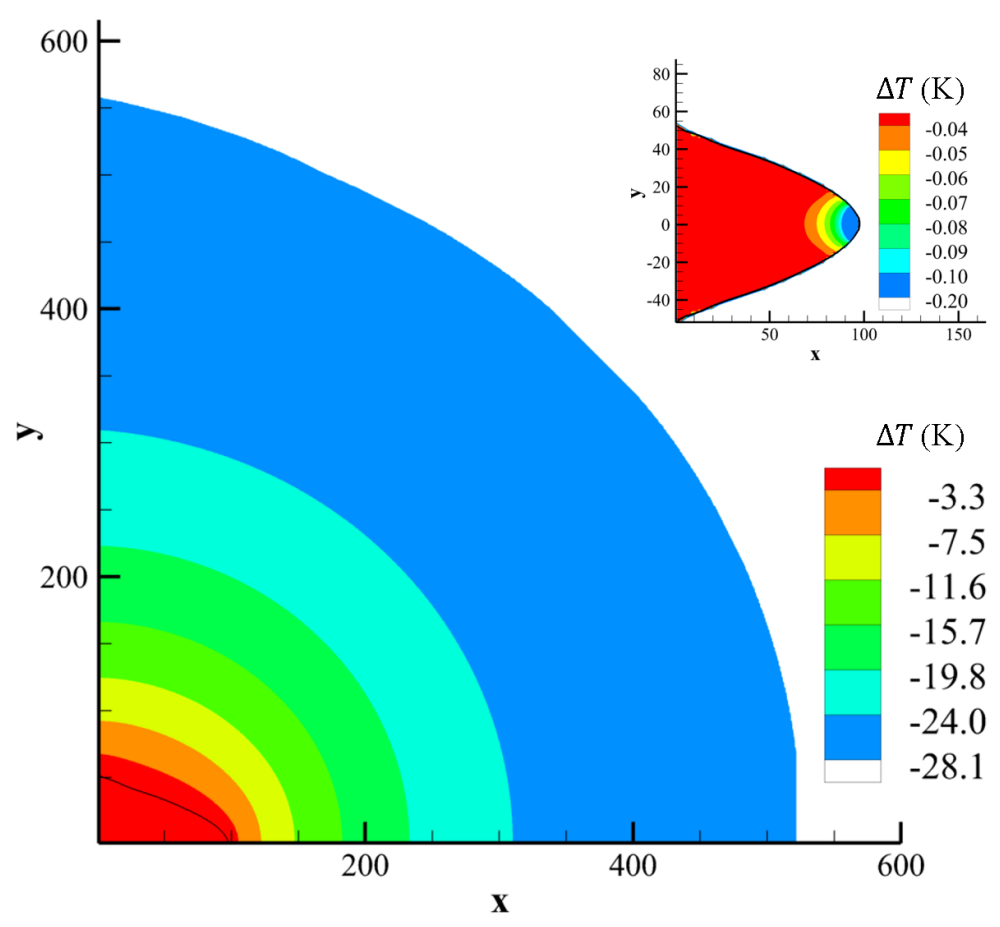

Figure 4. Thermal field for low, $28 \mathrm{~K}$, undercooling.

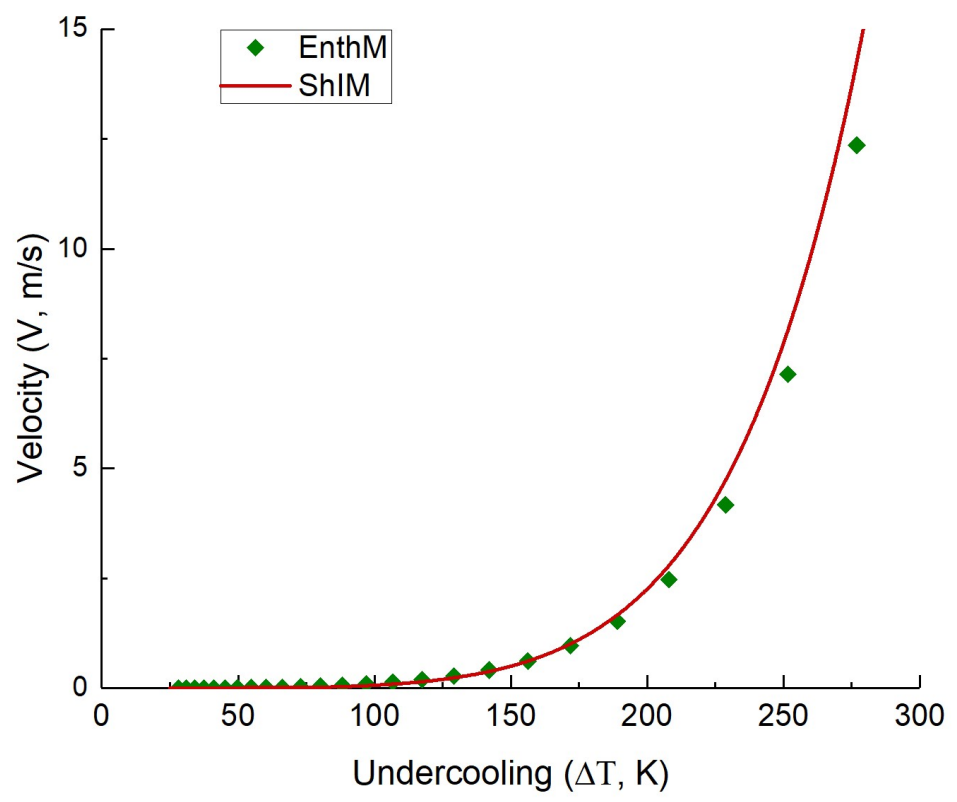

Figure 5. Dendrite tip velocity versus the melt undercooling for pure Nickel (physical parameters are listed in table 1 ). 


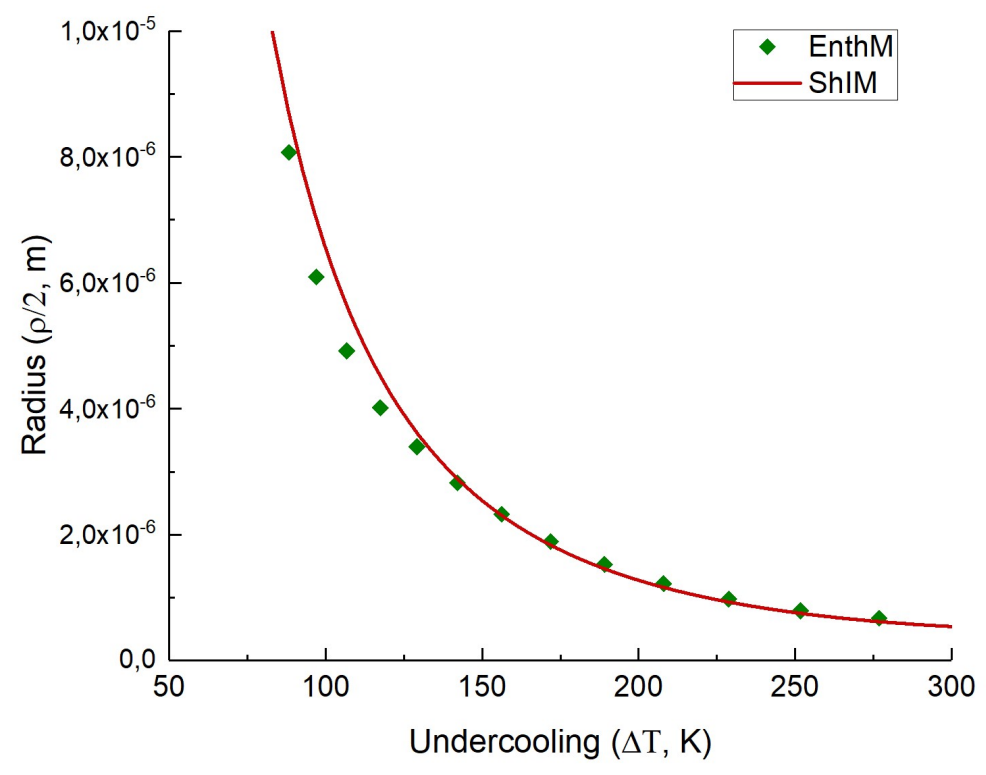

Figure 6. Dendrite tip radius versus the melt undercooling for pure Nickel (physical parameters are listed in table 1).

the undercooling balance (4) and the stability criterion (7) for pure nickel with the crystal symmetry $n=4$. Comparing the theory with computational simulations we see that both approaches are in good agreement in a broad range of melt undercooling (growth Péclet number). For example, a moderately low undercooling $\Delta T \approx 65 \mathrm{~K}$ gives the growth Péclet number $P_{g} \approx 0.008$ whereas the larger undercooling $\Delta T \approx 334$ $\mathrm{K}$ leads to an increased Péclet number $P_{g} \approx 0.66$. The last estimate means that the contribution $a_{1} \sqrt{\beta} P_{g}$ in (7) becomes of the order of 0.1. It gives a 20 percent correction to the denominator of the stability criterion (7) as compared with unity (with the lowvelocity stability criterion when the right-hand side of expression (7) is just $\sigma_{0} \beta^{7 / 4}$ ). More specifically, this definition of the stability criterion (7) describes the whole range of undercooling (all computationally achieved Péclet numbers) for the crystallization of single-component melts.

\section{Conclusion}

In summary, the sharp interface model based on the selection of stable mode for dendritic growth at the arbitrary Péclet numbers is compared with the computational modeling carried out using the enthalpy method. The comparison made for the dendrite tip velocity and dendrite tip radius for arbitrary Péclet number shows that the two-dimensional theory under consideration works well in the whole range of melt undercooling (Péclet numbers) and is consistent with the numerical enthalpy method. An excellent match between the numerical model and analytical solution was achieved. Our results highlight that the advanced stability criteria are necessary to 
Table 1. Physical and numerical parameters used in the present analytical calculations and numerical simulations for the pure Nickel. Here: ShIM means the sharp interface model and EnthM means the enthalpy method.

\begin{tabular}{llll}
\hline \hline Parameter & Symbol & ShIM & EnthM \\
\hline \hline Melting temperature $(\mathrm{K})$ & $T_{m}$ & 1728 & 1728 \\
Adiabatic temperature $(\mathrm{K})$ & $T_{Q}$ & 435 & 435 \\
Thermal diffusivity $\left(\cdot 10^{-5} \mathrm{~m}^{2} / \mathrm{s}\right)$ & $D_{T}$ & 1 & 1 \\
Density $\left(\cdot 10^{3} \mathrm{~kg} / \mathrm{m}^{3}\right)$ & $\rho_{N i}$ & 7,9 & 7,9 \\
Anisotropy strength & $\beta$ & 0.018 & 0.018 \\
Capillary length $\left(\cdot 10^{-10} \mathrm{~m}\right)$ & $d_{0}$ & 4 & 4 \\
Selection parameter & $\sigma_{0}$ & 0.05 & - \\
Selection parameter & $a_{1}$ & 1.9 & - \\
\hline
\end{tabular}

accurately predict the behavior of governing dependencies within a broad range of melt undercooling. On the computational side, this work also shows that the enthalpy method is viable for these fundamental studies, provided approaches are taken to mitigate against narrow-band feature errors, in this case by using an adaptive cell size method. As the enthalpy formulation has no explicit dependence on the interface thickness, it may have many advantages over traditional phase-field methods especially in a very low undercooled region where length scale disparities between the interface thickness, dendrite size, and thermal boundary layer become large.

Concluding this section, let us underline the main theoretical assumptions and future directions of the present study. In this work, it has been assumed that the dendrites are two-dimensional and they grow in a single-component melts in the absence of convection. Therefore an important task is to extend the theory and simulations to dendritic growth in three dimensions, as well as to investigate the effects of impurities and melt convection. This will allow for a direct comparison of the numerical model and analytic solution to experimental results. An extension to the case of a rapid crystallization scenario is also of fundamental significance. This will require using the generalized stability (solvability) criterion recently derived in ref. [53].

\section{Acknowledgments}

This work was supported by the Russian Science Foundation (grant No. 16-11-10095).

\section{References}

[1] Feuerbacher B 1989 Mater. Sci. Eng. Rep. 401

[2] Herlach D M 1994 Mater. Sci. Eng. Rep. 12177

[3] Biloni H and Boettinger W J 1996 Physical Metallurgy (Elsevier Science Publishers) vol. I 669

[4] Walker J L 1964 in Principles of Solidification (ed. Chalmers B) 4 (Wiley, New York) 122

[5] Colligan G A and Bayles B J 1962 Acta Metall. 10895 
[6] Eckler K, Hamerton R G, Herlach D and Greer A L 1991 Mater. Sci. Eng. A 133730

[7] Wu Y, Piccone T J, Shiohara Y and Flemings M C Metall. Mater. Trans. A 18915

[8] Matson D M 1998 in Solidification (eds. Marsh S P, Dantzig J A, Trivedi R, Hofmeister W, Chu $M G$, Lavernia $E J$ and Chun $J-H$ ) (The Mineral, Metals and Materials Society, Warrendale, PA) 233

[9] Coriell S R and Turnbull D 1982 Acta Metall. 302135

[10] Eckler K, Cochrane R F, Herlach D, Feuerbacher B and Jurisch M 1992 Phys. Rev. B 455019

[11] Eckler K, Herlach D and Aziz M 1994 Acta Metall. Mater. 42975

[12] Eckler K and Herlach D 1994 Mater. Sci. Eng. A 178159

[13] Schwarz M, Karma A, Eckler K and Herlach D M 1994 Phys. Rev. Lett. 731380

[14] Schwarz M and Herlach D M 1997 Mater. Sci. Eng. A 226-228 420

[15] Arnold C B, Aziz M J, Schwarz M and Herlach D M 1999 Phys. Rev. B 59334

[16] Barth M, Holland-Moritz D, Herlach D M, Matson D M and Flemings M C 1999 in: Solidification, edited by Hofmeister $W H$, Rogers $J$ R, Singh $N$ B, Marsh $S$ P and Vorhees $P$ (The Minerals, Metals and Materials Society, Warrendale, PA) 83

[17] Schenk T, Holland-Moritz D, Simonet V, Bellissent R and Herlach D M 2002 Phys. Rev. Lett. 89 075507

[18] Holland-Moritz D 1998 in: Solidification of Containerless Undercooled Melts, edited by Herlach D $M$ (Weinheim: Wiley) 69

[19] Funke O, Phanikumar G, Galenko P K, Chernova L, Reutzel S, Kolbe M and Herlach D M 2006 J. Cryst. Growth 29711

[20] Alexandrov D V, Galenko P K 2017 J. Phys. Chem. Solids 10898

[21] Gao J, Han M, Kao A, Pericleous K, Alexandrov D V and Galenko P K 2016 Acta Mater. 103 184

[22] Gao J, Kao A, Bojarevics V, Pericleous K, Galenko P K and Alexandrov D V 2017 J. Crys. Growth 47166

[23] Zykova-Timan T, Rozas R E , Horbach J and Binder K 2009 J. Phys.: Condens. Matter. 21464102

[24] Bokeloh J and Wilde G 2014 JOM 661512

[25] Hoyt J J, Sadigh B, Asta M and Foiles S M 1999 Acta Mater. 473181

[26] Mendelev M I, Rahman M J, Hoyt J J and Asta M 2010 Model. Simul. Mater. Sci. Eng 18074002

[27] Bragard J, Karma A, Lee Y H and Plapp M 2002 J. Colloid Interface Sci. 10121

[28] Hoyt J J, Asta M and Karma A 2003 Mater. Sci. Eng. R 41121

[29] Salhoumi A and Galenko P K 2017 IOP Conf. Series: Materials Science and Engineering 192 012014

[30] Apel M and Steinbach I 2009 in: Phase Transformations in Multicomponent Melts edited by Herlach D M (Weinheim: Weily) 373

[31] Nestler B, Danilov D and Galenko P K 2005 J. Comput. Phys. 207221

[32] Galenko P K, Reutzel S, Herlach D M, Danilov D and Nestler B 2007 Acta Mater. 556834

[33] Herlach D, Galenko P and Holland-Moritz D 2007 Metastable Solids from Undercooled Melts (Amsterdam: Elsevier)

[34] Alexandrov D V and Galenko P K 2014 Phys. Usp. 57771

[35] Barbieri A 1987 Phys. Rev. A 365353

[36] Barbieri A and Langer J S Phys. Rev. A 395314

[37] Alexandrov D V and Galenko P K Phys. Chem. Chem. Phys. 1719149

[38] Voller V 2008 Int. J. Heat Mass Trans. 51823

[39] Kao A and Pericleous K 2011 JACT 6173

[40] Alexandrov D V and Galenko P K 2013 Phys. Rev. E 87062403

[41] Alexandrov D V, Galenko P K and Toropova L V, 2018 Phil. Trans. R. Soc. A 37620170215

[42] Pelcé P and Bensimon D 1984 Nucl. Phys. B 2259

[43] Pelcé P 1988 Dynamics of Curved Fronts (Boston, MA: Academic Press)

[44] Bouissou Ph and Pelcé P 1989 Phys. Rev. A 406673 
[45] Alexandrov D V and Galenko P K 2018 J. Phys.: Condens. Matter 30105702

[46] Langer J S and Hong D C 1986 Phys. Rev. A 341462

[47] Brener E A and Mel'nikov V A 1991 Adv. Phys. 4053

[48] Kessler D A, Koplik J and Levine H 1988 Adv. Phys. 37255

[49] Tacke K-H 1990 in: K.-H. Hofmann, J. Sprekels (Eds.), Application of finite difference enthalpy methods to dendritic growth, free boundary problems: theory and applications, Longman Sci. Tech., Essex

[50] Tacke K-H and Harnisch A 1991 Proceedings of the International Conference on Computational Modeling of Free and Moving Boundary Problems, WIT press, Southampton.

[51] Karma A and Rappel W-J 1998 Phys. Rev. E 574323

[52] Bollada P C Jimack P K and Mullis A M 2018 Comp. Mat. Sci. 151338

[53] Alexandrov D V and Galenko P K 2019 IUTAM Symposium on Recent Advances in Moving Boundary Problems in Mechanics (IUTAM Bookseries, Springer) 34203 